13

\title{
Электронные и оптические свойства нанопленок $\mathrm{NiSi}_{2} / \mathrm{Si}$
}

\author{
() Б.Е. Умирзаков, Д.А. Ташмухамедова, А.К. Ташатов, Н.М. Мустафоева
}

Ташкентский государственный технический университет, 100095 Ташкент, Узбекистан

e-mail: ftmet@rambler.ru

Поступило в Редакцию 16 мая 2018 г.

В окончательной редакции 2 октября 2018 г.

Принято к публикации 14 ноября 2018 г.

Определены оптимальные условия ионной имплантации и последующего отжига для получения наноразмерных пленок $\mathrm{NiSi}_{2} / \mathrm{Si}$ (111) с толщиной 3.0-6.0nm. Показано, что зонно-энергетические параметры и оптические свойства, характерные для толстых пленок $\mathrm{NiSi}_{2}$, начинают устанавливаться с $d=5.0-6.0 \mathrm{~nm}$.

DOI: 10.21883/JTF.2019.05.47481.192-18

Пленки $\mathrm{CoSi}_{2}$ и $\mathrm{NiSi}_{2}$, полученные методами молекулярно-лучевой эпитаксии и твердофазной эпитаксии, широко используются в создании МДП- и ПДП-структур, транзисторов с проницаемой и металлической базами, барьерных слоев и омических контактов. Поэтому получению и изучению состава, структуры и свойств тонких пленок силицидов, особенно $\mathrm{CoSi}_{2}$, посвящено большое число работ [1-7]. В этих системах толщина пленки силицидов составляет не менее 30-50 nm. Уменьшение толщины этих пленок способствует созданию сверхвысокочастотных транзисторов и интегральных схем, работающих с предельной частотой $>100 \mathrm{GHz}$. Одним из перспективных методов получения нанокристаллов и нанопленок с толщиной $d<5-10 \mathrm{~nm}$ на поверхности полупроводников является низкоэнергетическая ионная имплантация [8-11]. В частности, в $[10,11]$ имплантацией ионов $\mathrm{Co}^{+}$в сочетании с отжигом на поверхности и в приповерхностном слое $\mathrm{Si}$ получены наноструктуры и нанопленки $\mathrm{CoSi}_{2}$. Изучены их состав, структура, электронные свойства и выяснены основные механизмы формирования слоев $\mathrm{CoSi}_{2}$.

В настоящей работе впервые исследованы основные физические свойства наноразмерных структур $\mathrm{NiSi}_{2}$, созданных на поверхности $\mathrm{Si}$ методом низкоэнергетической ионной имплантации.

\section{Методика экспериментов}

Объектами исследования являлись монокристаллические образцы $n$-типа $\mathrm{Si}$ (111). Перед ионной имплантацией кремниевые образцы очищались прогревом при вакууме $P=10^{-7} \mathrm{~Pa}$ сначала длительно $(2-3 \mathrm{~h})$ при $T=1100 \mathrm{~K}$ и затем кратковременно при $T=1400 \mathrm{~K}$. Имплантация ионов $\mathrm{Ni}$ проводилась с энергией $E_{0}=1-5 \mathrm{keV}$ при дозе насыщения $(D=$ $\left.=(6-8) \cdot 10^{16} \mathrm{~cm}^{-2}\right)$. Элементный и химический составы поверхности определяли методом оже-электронной спектроскопии (ОЭС), электронная структура (параметры энергетических зон, плотность состояния валентных электронов) - методами ультрафиолетовой фотоэлектронной спектроскопии (УФЭС) и снятия зависимости $I(h v)$, где $I-$ интенсивность света, проходящая через образец, $h$ - энергия фотонов $(h v=0.2-1.5 \mathrm{eV})$. Профили распределения атомов по глубине определялись методом ОЭС в сочетании с травлением поверхности ионами $\mathrm{Ar}^{+}$. Более подробное описание методики эксперимента приведено в [10].

\section{Результаты экспериментов и их обсуждение}

На рис. 1 приведены концентрационные профили распределения $\mathrm{Ni}$ по глубине $\mathrm{Si}$, имплантированного ионами $\mathrm{Ni}^{+}$с $E_{0}=1 \mathrm{keV}$ при дозе насыщения $D=6 \cdot 10^{16} \mathrm{~cm}^{-2}$ измеренных до и после прогрева при $T=900 \mathrm{~K}$. Видно, что после ионной имплантации (кривая 1) концентрация $\mathrm{Ni}$ на поверхности составляет 45-50 at.\% и до глубины $3.0-3.5 \mathrm{~nm}$ заметно не меняется, затем монотонно уменьшается и при

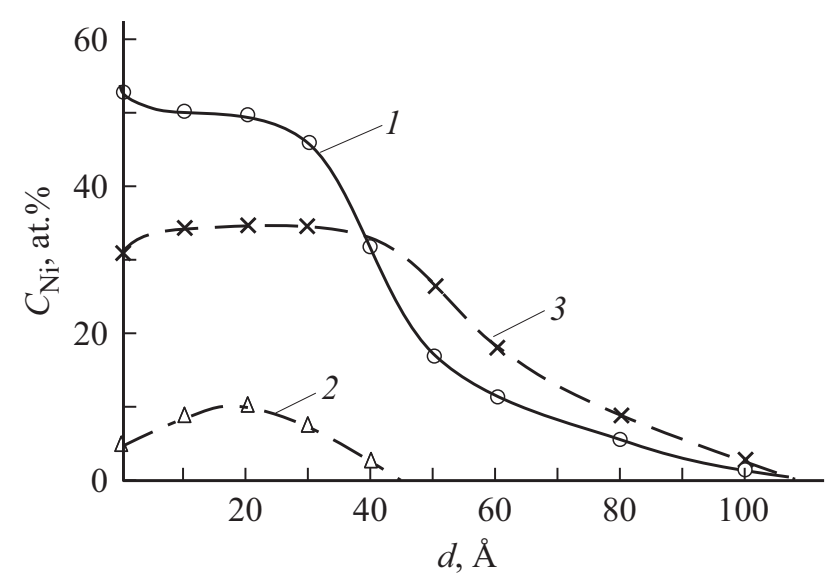

Рис. 1. Профили распределения концентрации Ni (кривая 1) и $\mathrm{Ni}+\mathrm{Si}$ (кривая 2) по глубине $\mathrm{Si}$, имплантированного ионами Ni c $E_{0}=1 \mathrm{keV}$ при дозе $D=6 \cdot 10^{16} \mathrm{~cm}^{-2}$ до $(1,2)$ и после прогрева при $T=900 \mathrm{~K}(3)$. 


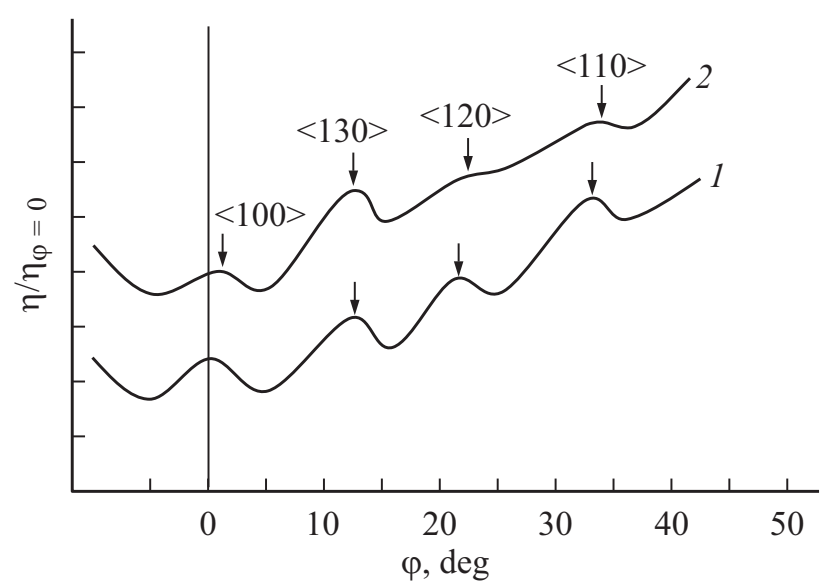

Рис. 2. Зависимости $\eta / \eta_{\varphi=0}$ от угла падения электронов $E_{e}=2 \mathrm{keV} .1-\mathrm{Si} ; 2-\mathrm{NiSi}_{2} / \mathrm{Si}$.

$d=9.0-10.0 \mathrm{~nm}$ равняется нулю (в пределах чувствительности ОЭС). При этом приповерхностные слои полностью разупорядочиваются. В ионно-легированном слое содержатся не связанные атомы $\mathrm{Si}$ и $\mathrm{Ni}$, а также соединения типа $\mathrm{Ni}+\mathrm{Si}$. Мы попытались оценить концентрацию этих компонент в различных глубинах ионноимплантированного слоя по относительному изменению положения и интенсивности пиков $\mathrm{L}_{23} \mathrm{VV}$ и $\mathrm{M}_{3} \mathrm{VV}$. Из рис. 1 кривой 2 видно, что зависимость $C_{\mathrm{Ni}+\mathrm{Si}}(d)$ проходит через максимум в области $d=1.5-2.0 \mathrm{~nm}$. По-видимому, образование силицидов металла имеет более высокую вероятность не на поверхности, а внутри ионно-легированного слоя.

После прогрева при $T=900 \mathrm{~K}$ в течение $30 \mathrm{~min}$ концентрация $\mathrm{Ni}$ на поверхностных слоях уменьшается и формируется однородный эпитаксиальный слой стехиометрического состава $\mathrm{NiSi}_{2}$ (111) с толщиной $\sim 3.5-4.0 \mathrm{~nm}$ (кривая 3). Анализ спектров ОЭС показывает, что как в процессе ионной имплантации, так и последующего высокотемпературного отжига преимущественно образуются соединения типа $\mathrm{NiSi}_{2}$. По-видимому, это обусловлено тем, что $\mathrm{NiSi}_{2}$ и $\mathrm{Si}$ имеют одинаковую кристаллическую структуру и постоянные их решетки хорошо совпадают друг с другом (несоответствие $\sim 0.4 \%$ ). Другие силициды $\mathrm{Ni}$ либо не кристаллизуются в кубическую решетку, либо кристаллизуются с постоянной решетки, отличающейся от „а “ кремния [12].

Между пленками $\mathrm{NiSi}_{2}$ (111) и $\mathrm{Si}(111)$ формируется переходной слой с толщиной 4.0-5.0 nm. Однако до сих пор не имеются достоверные сведения, как изменяется состав силицида и как изменяется тип и параметры решетки этих силицидов в переходном слое. Для оценки кристалличности и эпитаксиальности пленок $\mathrm{NiSi}_{2}$ и переходного слоя нами изучены угловые зависимости коэффициентов вторичной электронной эмиссии. На рис. 2 приведены зависимости значений коэффициента неупругоотраженных электронов (НОЭ) $\eta$ от угла падения $\varphi$ первичного пучка для чистого $\mathrm{Si}$ и $\mathrm{NiSi}_{2} / \mathrm{Si}$ (111), снятых при энергии первичных электронов $E_{p}=1200 \mathrm{eV}$. Угол $\varphi$ определялся относительно нормали образца. При $E_{p}=1200 \mathrm{eV}$ глубина выхода НОЭ $\lambda_{\eta}$ для $\varphi=0$ составляет $\sim 35.0-40.0 \mathrm{~nm}$. При увеличении $\varphi$ до 50-60 ${ }^{\circ}$ значение $\lambda_{\eta}$ может уменьшаться $1.5-2$ раза, т.е. HОЭ-электроны эмиттируют как из $\mathrm{NiSi}_{2}$ и переходного слоя, так и из подложки. Из рис. 2 видно, что для этих образцов с ростом $\varphi$ наблюдается немонотонный рост значений $\eta$, т.е. на зависимостях $\eta(\varphi)$ обнаруживаются пики, положение которых связано с определенными кристаллическими направлениями. $\eta_{\varphi=0}-$ значение $\eta$ при $\varphi=0$. Сравнение кривых 1 и 2 показывает, что угловое положение основных пиков $\mathrm{Si}$ и $\mathrm{NiSi}_{2} / \mathrm{Si}$ хорошо совпадает друг с другом, т. е. тип и постоянные решетки $\mathrm{NiSi}_{2}$ и переходного слоя практически идентичны с таковыми для $\mathrm{Si}$. Что касается изменения состава силицида при изменении концентрации $\mathrm{Ni}$ от $30-35$ at.\% до нуля пока нет однозначного ответа. Можно полагать, что в переходном слое в узлах решетки в зависимости от $C_{\mathrm{Ni}}$ происходит частичная замена $\mathrm{Si}$ атомами Ni.

На рис. 3 приведены фотоэлектронные спектры $\mathrm{Si}$ и $\mathrm{Si}$ с нанопленкой $\mathrm{NiSi}_{2}$, снятые при $h v=10.8 \mathrm{eV}$. По оси абсцисс отложена энергия связи $E_{r e l}$ электронов, отсчитанная относительно уровня Ферми $E_{\mathrm{F}}$. Видно, что на кривых энергетического распределения фотоэлектронов 1 и 2 форма и положения пиков резко отличаются друг от друга, т.е. образование силицида приводит к существенному изменению плотности состояния валентных электронов кремния. Основные особенности, наблюдаемые на спектре $\mathrm{Si}$, можно связать с возбуждением электронов из поверхностных состояний (ПС), а также $3 p-$ и $3 s$-состояниями валентных электронов. В случае пленки $\mathrm{NiSi}_{2}$ явно выраженные особенности обнаруживаются при энергиях $-1.0,-2.7$ и $-4.0 \mathrm{eV}$.

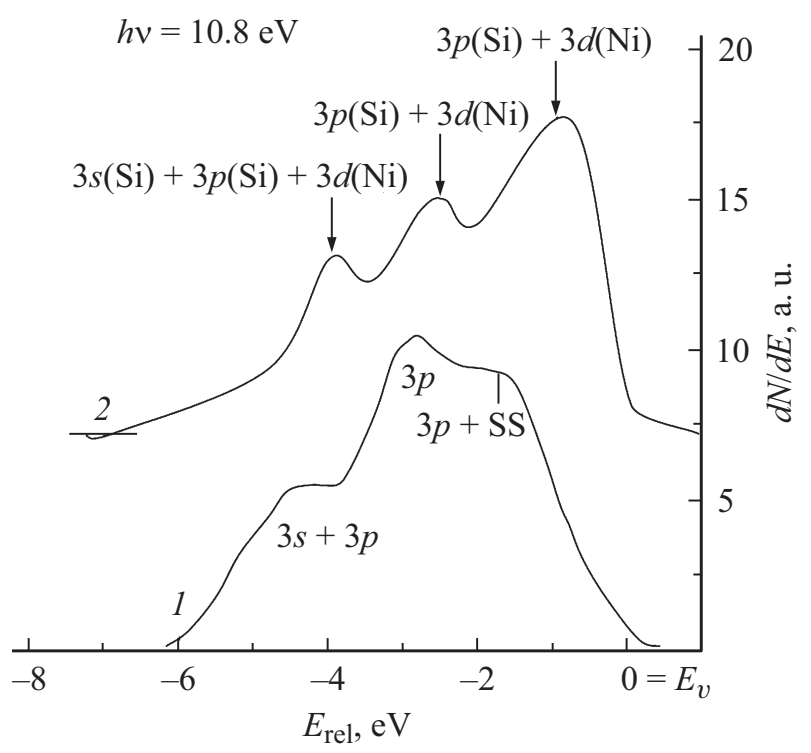

Рис. 3. УФЭС для $1-\mathrm{Si}(111) ; 2-$ нанопленки $\mathrm{NiSi}_{2} / \mathrm{Si}$ (111) c $d=3.5-4.0 \mathrm{~nm}$. 
Зонно-энергетические, эмиссионные и оптические параметры $\mathrm{Si}(111)$ и пленки $\mathrm{NiSi}_{2} / \mathrm{Si}(111)$

\begin{tabular}{c|c|c|c|c|c|c|c|c}
\hline Образец & $d, \mathrm{~nm}$ & $\varphi, \mathrm{eV}$ & $\Phi, \mathrm{eV}$ & $E_{g}, \mathrm{eV}$ & $\chi, \mathrm{eV}$ & $\sigma_{m}$ & $Y$ & $\rho, \mu \Omega \cdot \mathrm{cm}$ \\
\hline $\mathrm{Si}(111)$ & 0 & 4.7 & 5.1 & 1.1 & 4 & 1.1 & $2 \cdot 10^{-4}$ & $5 \cdot 10^{6}$ \\
\hline & 3.0 & 4.3 & 4 & 0.6 & 3.4 & 1.5 & $4 \cdot 10^{-4}$ & 80 \\
$\mathrm{NiSi}_{2} / \mathrm{Si}(111)$ & 6.0 & - & 4 & 0.5 & 3.5 & 1.6 & $4 \cdot 10^{-4}$ & 60 \\
& 50.0 & 4.2 & 4 & 0.5 & 3.5 & 1.6 & - & 55
\end{tabular}

Эти особенности, по-видимому, формируются вследствие гибридизации $3 d\left(3 d_{5 / 2}\right.$ и $\left.3 d_{3 / 2}\right)$ состояния $\mathrm{Ni} \mathrm{c}$ $3 p-$ и $3 s$-состояниями $\mathrm{Si}$.

Аналогичные исследования проводились и для пленок $\mathrm{NiSi}_{2} / \mathrm{Si}$, полученных имплантацией ионов $\mathrm{Ni} \mathrm{c} E_{0}=3$ и $5 \mathrm{keV}$. При этом после прогрева формировались пленки $\mathrm{NiSi}_{2}$ с толщиной $4.5-5.0$ и $6.0-6.5 \mathrm{~nm}$ соответственно. $\mathrm{B}$ таблице приведены некоторые физические параметры $\mathrm{Si}$ и пленки $\mathrm{NiSi}_{2} / \mathrm{Si}$ с толщиной $d=3.0$ и $6.0 \mathrm{~nm}$, где $\varphi$ и $\Phi$ - термо- и фотоэлектронная работа выхода, $E_{g}-$ ширина запрещенной зоны, $\chi-$ сродство к электрону, $\sigma_{m}$ - максимальное значение коэффициента ВЭЭ, $Y$ квантовый выход фотоэлектронов, $\rho$ - удельное сопротивление. Для сравнения там же приведены параметры для толстой пленки $\mathrm{NiSi}_{2}$ с толщиной $50.0 \mathrm{~nm}$, полученной методом твердофазной эпитаксии. Из таблицы видно, что ширина запрещенной зоны $\mathrm{NiSi}_{2}$ с $d=3.0 \mathrm{~nm}$ составляет $0.6 \mathrm{eV}$ и обладает свойствами, близкими к металлам $(\rho=80 \mu \Omega \cdot \mathrm{cm})$. При этом значение $\sigma_{m}$ и $Y$ увеличивается до 1.5 и 2 раза. По-видимому, эмиссионная эффективность слоев $\mathrm{NiSi}_{2}$ немного больше, чем эффективности слоев $\mathrm{Si}$, что может быть связано с заметным отличием атомной плотности $\mathrm{NiSi}_{2}\left(\sim 4.5 \mathrm{~g} / \mathrm{cm}^{3}\right)$ от плотности $\mathrm{Si}\left(2.42 \mathrm{~g} / \mathrm{cm}^{3}\right)$. Электронные и оптические свойства пленок $\mathrm{NiSi}_{2}$ с $d=6.0 \mathrm{~nm}$ существенно не отличались от таковых для толстой пленки.

\section{Заключение}

Методом низкоэнергетической $\left(E_{0}=1-5 \mathrm{keV}\right)$ имплантации ионов $\mathrm{Ni}$ в сочетании с отжигом получены однородные эпитаксиальные нанопленки $\mathrm{NiSi}_{2}$ с толщиной $\sim 3.0-6.0 \mathrm{~nm}$. Исследованы зонно-энергетические параметры, плотности электронных состояний, эмиссионные и оптические параметры системы $\mathrm{NiSi}_{2} / \mathrm{Si}$ (111). Показано, что ширина запрещенной зоны нанопленок $\mathrm{NiSi}_{2}$ составляет $\sim 0.5-0.6 \mathrm{eV}$, квантовый выход фотоэлектронов $\sim 4 \cdot 10^{-4}$, удельное сопротивление $60-80 \mu \Omega \cdot \mathrm{cm}$.

\section{Список литературы}

[1] Алексеев А.А., Олянич Д.А., Утас Т.В., Котляр В.Г., Зотов А.В., Саранин А.А. // ЖТФ. 2015. Т. 85. Вып. 10. C. $94-100$.

[2] Kim K.J., Kang T.-H., Kim K.-W., Shin H.-J., Kim B. // Appl. Surf. Sci. 2000. Vol. 161. P. 268-275.
[3] Starke U., Schardt J., Weiß W., Rangelov G., Fauster T.H., Heinz K. // Surf. Rev. Lett. 1998. Vol. 5. P. 139-144.

[4] Гомоюнова М.В., Пронин И.И., Галль Н.Р., Молоцов С.Л., Вялых Д.В. // ФТТ. 2003. Т. 45. Вып. 8. С. 1519.

[5] Colinge J.P. // Material Research Society Proceedings. 1985. Vol. 35. P. 653. http://dx.doi.org/doi 10.1557/ PROC-35-653

[6] Алтухов А.А., Жирнов В.В. Анализ морфологии и стехиометрии пленок $\mathrm{CoSi} / \mathrm{Si}(100)$, полученных методами ТФЭ и РЭ // Материалы II-го Всесоюзного межотраслевого совещания „Тонкие пленки в электронике“: Москва-Ижевск. 1991. С. 15.

[7] Рудаков В.И., Денисенко Ю.И., Наумов В.В., Симакин С.Г. // Микроэлектроника. 2011. Т. 40. № 6. С. 424-429.

[8] Мурадкабилов Д.М., Ташмухамедова Д.А., Умирзаков Б.Е. // Поверхность. Рентгеновские, синхротронные и нейтронные исследования. 2013. № 10. С. 58-62. [Muradkabilov D.M., Tashmukhamedova D.A., Umirzakov B.E. // J. Surf. Investigation. X-ray, Synchrotron and Neutron Techniques. 2013. Vol. 7. N 5. P. 967-971.]

[9] Умирзаков Б.Е., Ташмухамедова Д.А., Рузибаева М.К., Ташатов А.К., Донаев С.Б., Мавлянов Б.Б. // ЖТФ. 2013. Т. 83. Вып. 9. С. 146-149. [Umirzakov B.E., Tashmukhamedova D.A., Ruzibaeva M.K., Tashatov A.K., Donaev S.B., Mavlyanov B.B. // Techn. Phys. 2013. Vol. 58. N 9. P. 1383-1386.]

[10] Umirzakov B.E., Pugacheva T.S., Tashatov A.K., Tashmukhamedova D.A. // NIM-B. 2000. Vol. 166-167. P. 572-576.

[11] Умирзаков Б.Е., Ташмухамедова Д.А., Мурадкабилов Д.М., Болтаев Х.Х. // ЖТФ. 2013. Т. 83. Вып. 6. C. $66-70$ [Umirzakov B.E., Tashmukhamedova D.A., Muradkabilov D.M., Boltaev K.K. // Technical Physics, 2013, Vol. 58, N 6, p. 841-844.]

[12] Самсонов Т.В., Дворина Л.А., Рудь Б.М. Силициды. М.: Металлургия, 1979. 372 с. 